\title{
A THEOREM FOR KERNEL FUNCTIONS
}

PHILIP DAVIS AND HENRY POLLAK

Let $B$ be a domain lying in the complex $z$ plane and $K_{B}(z, \bar{t})$ its kernel function. A number of relationships exist between the kernel and the geometric properties of the domain. (See, for example, [1].) ${ }^{1}$ It is the purpose of the present note to relate the successive derivatives of the kernel with the domain $B$.

If $z$ is interior to $B$, we shall denote by $r_{B}(z)$ the shortest distance from the point $z$ to the boundary of $B$. Furthermore, we introduce the abbreviation

$$
K_{B}^{(m, n)}(z, \bar{t}) \equiv \frac{\partial^{m+n}}{\partial z^{m} \partial \bar{t}^{n}} K_{B}(z, \bar{t}) .
$$

THEOREM 1. Let the domain $B$ be such that at every boundary point $z_{1}$ there exists ${ }^{2}$ a circle exterior to $B$ and passing through $z_{1}$. Then,

$$
1 / r_{B}(z)=\limsup _{n \rightarrow \infty}(e / n)\left[K_{B}^{(n, n)}(z, \bar{z})\right]^{1 / 2 n} .
$$

Proof. It obviously suffices to prove the theorem for $z=0$, it being supposed that $B$ contains this point in its interior.

Let $r=r_{B}(0)$, and suppose that $z_{1}$ is a point which simultaneously lies on the boundary of $B$ and on the circle $|z|=r$. In the bicylinder $|z| \leqq r^{*}<r,|t| \leqq r^{*}<r, K_{B}(z, \bar{t})$ is an analytic function of the two complex variables $z$ and $\bar{t}$ and hence has an expansion of the form

$$
K_{B}(z, \bar{t})=\sum_{m, n=0}^{\infty} K_{B}^{(m, n)}(0,0) z^{m} \bar{t}^{n} / m ! n !
$$

converging absolutely and uniformly there. If, then, $\lambda$ is a real variable with $|\lambda|<r / r^{*}$, then

$$
K_{B}(\lambda z, \lambda \bar{t})=\sum_{m, n=0}^{\infty} K_{B}^{(m, n)}(0,0) z^{m^{n} \bar{t}^{n} \lambda^{m+n} / m ! n ! .}
$$

With $\lambda$ in the above range, (4) converges absolutely and uniformly in $|z|,|t| \leqq r^{*}$. The circle $|z|=r^{*}$ will be designated by $C^{*}$. Thus, for $|\lambda|<r / r^{*}$,

Received by the editors September 25, 1950.

${ }^{1}$ Number 1 in brackets refers to the bibliography at the end of the paper.

2 The theorem may be established for a wider class of boundaries. Moreover, a similar result holds for several complex variables. 


$$
\begin{aligned}
F(\lambda) & \equiv \iint_{C^{*}} K_{B}(\lambda z, \lambda \bar{z}) d x d y \\
& =\sum_{m, n=0}^{\infty} K_{B}^{(m, n)}(0,0) \lambda^{m+n} / m ! n ! \iint_{C^{*}} z^{m \bar{z}^{n}} d x d y \\
& =\sum_{n=0}^{\infty} K_{B}^{(n, n)}(0,0) r^{* 2 n+2} \lambda^{2 n} /(n !)(n+1) !
\end{aligned}
$$

The function $F(\lambda)$ is therefore analytic for $|\lambda|<r / r^{*}$.

We shall prove, moreover, that $F(\lambda)$ has a singularity at the point $\lambda=r / r^{*}$. If this is admitted momentarily, then by the familiar Cauchy-Hadamard formula for the radius of convergence of a power series, we shall have

$$
\begin{aligned}
r^{*} / r & =\limsup _{n \rightarrow \infty}\left[K_{B}^{(n, n)}(0,0) r^{* 2 n+2} / n !(n+1) !\right]^{1 / 2 n} \\
& =r^{*} \limsup _{n \rightarrow \infty}(e / n)\left[K_{B}^{(n, n)}(0,0)\right]^{1 / 2 n}
\end{aligned}
$$

from which (2) follows.

We shall show that

$$
\lim _{\lambda \rightarrow r / r^{*}-} F(\lambda)=\infty .
$$

Suppose that $C_{1}$ designates a circle of radius $r_{1}$ which is exterior to $B$ but whose circumference passes through $z_{1}$. Designate the exterior of the circle by $D$. By the monotonicity of the kernel function

$$
K_{B}(\lambda z, \lambda \bar{z}) \geqq K_{D}(\lambda z, \lambda \bar{z}) .
$$

Therefore,

$$
\begin{aligned}
F(\lambda) & \geqq \iint_{C^{*}} K_{D}(\lambda z, \lambda \bar{z}) d x d y \\
& =\lambda^{-2} \iint_{\lambda C^{*}} K_{D}(z, \bar{z}) d x d y \equiv I(\lambda) .
\end{aligned}
$$

In the above equation, $\lambda C^{*}$ designates the circle $|z| \leqq \lambda r^{*}$. To estimate the integral $I(\lambda)$, it is convenient to introduce new coordinates $z^{\prime}=x^{\prime}+i y^{\prime}$ as follows: $z_{1}$ shall be the new origin, and the center of $C_{1}$ shall lie on the negative $x^{\prime}$ axis. In the new system, $K_{D}$ is given by

$$
K_{D}\left(z^{\prime}, \bar{z}^{\prime}\right)=\frac{1}{\pi}\left(z^{\prime}+\bar{z}^{\prime}+\frac{z^{\prime} \bar{z}^{\prime}}{r_{1}}\right)^{-2}=\frac{1}{\pi}\left(2 x^{\prime}+\frac{x^{\prime 2}+y^{\prime 2}}{r_{1}}\right)^{-2} \text {. }
$$


For values of $\lambda$ sufficiently near to $r / r^{*}$, the ray $x^{\prime}=y^{\prime}$ will intersect the circle $\lambda C^{*}$ in two points whose abscissas $x_{1}^{\prime}(\lambda), x_{2}^{\prime}(\lambda)$ have the property

$$
\lim _{\lambda \rightarrow r / r^{*}-} x_{1}^{\prime}(\lambda)=0 ; \quad \lim _{\lambda \rightarrow r / r^{*}-} x_{2}^{\prime}(\lambda)=r .
$$

Designate by $T(\lambda)$ the trapezoid bounded by $y^{\prime}=0, y^{\prime}=x^{\prime}, x^{\prime}$ $=x_{i}^{\prime}(\lambda)(i=1,2) . T(\lambda)$ is contained in the circle $\lambda C^{*}$. Now,

$$
x^{\prime 2} K_{D}\left(z^{\prime}, \bar{z}^{\prime}\right)=\frac{1}{\pi}\left(2+x^{\prime} / r_{1}+y^{\prime 2} / x^{\prime} r_{1}\right)^{-2}
$$

so that if $\left(x^{\prime}, y^{\prime}\right)$ is confined to $T(\lambda)$, then $0 \leqq x^{\prime} \leqq r, 0 \leqq y^{\prime} \leqq r, 0 \leqq y^{\prime} / x^{\prime}$ $\leqq 1$, and hence

$$
x^{\prime 2} K_{D}\left(z^{\prime}, \bar{z}^{\prime}\right) \geqq \frac{1}{4 \pi}\left(1+\left(r / r_{1}\right)\right)^{-2}=k>0
$$

for $z^{\prime}$ in $T(\lambda)$. Combining our inequalities we have

$$
I(\lambda) \geqq \lambda^{-2} \iint_{T(\lambda)} k / x^{\prime 2} d x^{\prime} d y^{\prime}=\lambda^{-2} \log \left[x_{2}^{\prime}(\lambda) / x_{1}^{\prime}(\lambda)\right] .
$$

The assertion (7) now follows from (9) and (11), and the proof is complete.

As an application of Theorem 1, we show how, for the case of a simply-connected domain, the quantity $r_{B}(0)$ may be expressed in terms of the coefficients of the mapping function of the domain onto the unit circle.

TheOREM 2. Let $w(z)=a_{1} z+a_{2} z^{2}+\cdots$ map the simply connected domain $B$ onto the unit circle in the w-plane, $z=0$ corresponding to $w=0$. Then,

$$
1 / r=\limsup _{n \rightarrow \infty}\left\{\sum_{\nu=0}^{n}(\nu+1)\left|\sum_{j=\nu}^{n} \frac{(n-j+1)}{j !} a_{n-j+1}\left(\frac{d^{j} w^{\nu}}{d z^{j}}\right)_{0}\right|^{2}\right\}^{1 / 2 n} .
$$

Proof. If $C$ designates the unit circle in the $w$-plane, then by the conformal invariance of the kernel function we have

$$
K_{B}(z, \bar{z})=K_{C}(w, \bar{w}) \frac{d w}{d z}\left(\frac{d w}{d z}\right)^{-} .3
$$

Now,

${ }^{3} z^{-}=x-i y$ 


$$
\frac{\partial^{n}}{\partial z^{n}} K_{B}(z, \bar{z})=\sum_{j=0}^{n} C_{n, j}\left(\frac{d w}{d z}\right)^{-} \frac{\partial^{j} K_{C}(w, \bar{w})}{\partial z^{j}} \frac{d^{n-j+1} w}{d z^{n-j+1}}
$$

Furthermore,

$$
\begin{aligned}
& \frac{\partial^{n}}{\partial \bar{z}^{n}}\left(\left(\frac{d w}{d z}\right)-\frac{\partial^{j} K_{C}(w, \bar{w})}{\partial z^{j}}\right) \\
&=\sum_{p=0}^{n} C_{n, p} \frac{\partial^{j+p} K_{C}(w, w)}{\partial z^{j} \partial \bar{z}^{p}}\left(\frac{d^{n-p+1} w}{d z^{n-p+1}}\right)-
\end{aligned}
$$

so that

$$
\begin{aligned}
K_{B}^{(n, n)}(z, \bar{z}) & \\
& =\sum_{j=0, p=0}^{n} C_{n, j} C_{n, p} \frac{d^{n-j+1} w}{d z^{n-j+1}}\left(\frac{d^{n-p+1} w}{d z^{n-p+1}}\right)-\frac{\partial^{j+p} K_{C}(w, w)}{\partial z^{j} \partial \bar{z}^{p}} .
\end{aligned}
$$

Again,

$$
K_{C}(w, \bar{w})=\frac{1}{\pi} \sum_{\nu=0}^{\infty}(\nu+1) w^{\nu} \bar{w}^{\nu}
$$

Thus

$$
\frac{\partial^{j+p}}{\partial z^{j} \partial \bar{z}^{p}} K_{C}(w, \bar{w})=\frac{1}{\pi} \sum_{\nu=0}^{\infty}(\nu+1) \frac{d^{j} w^{\nu}}{d z^{j}}\left(\frac{d^{p} w^{\nu}}{d z^{p}}\right) \text { - }
$$

Hence

$$
\begin{aligned}
K_{B}^{(n, n)}(0,0) & =\frac{1}{\pi} \sum_{j, p=0}^{n} C_{n, j} C_{n, p}\left(\frac{d^{n-j+1} w}{d z^{n-j+1}}\right)_{0}\left(\frac{d^{n-p+1} w}{d z^{n-p+1}}\right)_{0}^{\min (p, j)}\left(\sum_{\nu=0}+1\right)\left(\frac{d^{j} w^{\nu}}{d z^{j}}\right)_{0}\left(\frac{d^{p} w^{\nu}}{d z^{p}}\right)_{0} \\
= & \frac{(n !)^{2}}{\pi} \sum_{\nu=0}^{n}(\nu+1) \sum_{j, p=0}^{n}(n-j+1)(n-p+1) a_{n-j+1}\left(a_{n-p+1}\right) \\
= & \left.\frac{(n !)^{2}}{\pi} \sum_{\nu=0}^{n}(\nu+1) \mid \sum_{j=\nu}^{n} \frac{(n-j+1)}{j !} \frac{1}{p !}\right)\left(\frac{d^{j} w^{\nu}}{d z^{j}}\right)_{0}\left(\frac{d^{p} w^{\nu}}{d z^{p}}\right)_{0}
\end{aligned}
$$


An application of Theorem 1 now yields (15).

\section{BIBLIOGRAPHY}

1. Stefan Bergman, The kernel function and conformal mapping, Mathematical Surveys, no. 5, New York, American Mathematical Society, 1950.

HARVARD UNIVERSITY

\section{REMARK ON THE HURWITZ ZETA FUNCTION}

T. M. APOSTOL

The Hurwitz zeta function, defined for $0<a \leqq 1, \Re(s)>1$, by

$$
\zeta(s, a)=\sum_{n=0}^{\infty} \frac{1}{(a+n)^{s}},
$$

is given [2] ${ }^{1}$ in the negative half-plane by means of

$$
\begin{aligned}
\zeta(1-s, a)= & \frac{2 \Gamma(s)}{(2 \pi)^{s}}\left(\cos \frac{\pi s}{2} \sum_{n=1}^{\infty} \frac{\cos (2 \pi a n)}{n^{s}}\right. \\
& \left.+\sin \frac{\pi s}{2} \sum_{n=1}^{\infty} \frac{\sin (2 \pi a n)}{n^{s}}\right) \\
= & \frac{2 \Gamma(s)}{(2 \pi)^{s}} \sum_{n=1}^{\infty} \frac{\cos (\pi s / 2-2 \pi a n)}{n^{s}} \quad(\Re(s)>1) .
\end{aligned}
$$

The functional equation for the Riemann zeta function is obtained from (2) upon setting $a=1$.

The more general function

$$
\phi(x, a, s)=\sum_{n=0}^{\infty} \frac{e^{2 \pi i n x}}{(a+n)^{s}}
$$

reduces to $\zeta(s, a)$ when $x$ is an integer. Lerch [1] derived the transformation formula

$$
\begin{aligned}
\phi(x, a, 1-s)=\frac{\Gamma(s)}{(2 \pi)^{\circ}}\left\{e^{\pi i(s / 2-2 a x)} \phi(-a, x, s)\right. \\
\left.+e^{\pi i(-s / 2+2 a(1-x))} \phi(a, 1-x, s)\right\}
\end{aligned}
$$

Presented to the Society, June 16, 1951; received by the editors August 24, 1950 and, in revised form, October 9, 1950.

${ }^{1}$ Numbers in brackets refer to the bibliography at the end of the paper. 